\title{
The receptor locus for Escherichia coli F4ab/F4ac in the pig maps distal to the MUC4-LMLN region
}

\section{Journal Article}

\section{Author(s):}

Rampoldi, Antonio; Jacobsen, Mette J.; Bertschinger, Hans U.; Joller, David; Bürgi, Ester; Vögeli, Peter; Andersson, Leif; Archibald, Alan L.; Fredholm, Merete; Jørgensen, Claus B.; Neuenschwander, Stefan

\section{Publication date:}

2011-02

\section{Permanent link:}

https://doi.org/10.3929/ethz-b-000026882

\section{Rights / license:}

In Copyright - Non-Commercial Use Permitted

\section{Originally published in:}

Mammalian Genome 22(1-2), https://doi.org/10.1007/s00335-010-9305-3 


\title{
The receptor locus for Escherichia coli F4ab/F4ac in the pig maps distal to the MUC4-LMLN region
}

\author{
Antonio Rampoldi • Mette J. Jacobsen · Hans U. Bertschinger • David Joller • \\ Esther Bürgi · Peter Vögeli • Leif Andersson • Alan L. Archibald • \\ Merete Fredholm · Claus B. Jørgensen · Stefan Neuenschwander
}

Received: 2 July 2010/ Accepted: 3 November 2010/Published online: 7 December 2010

(C) Springer Science+Business Media, LLC 2010

\begin{abstract}
Enterotoxigenic Escherichia coli (ETEC) with fimbriae of the F4 family are one of the major causes of diarrhea and death among neonatal and young piglets. Bacteria use the F4 fimbriae to adhere to specific receptors expressed on the surface of the enterocytes. F4 fimbriae exist in three different antigenic variants, F4ab, F4ac, and F4ad, of which F4ac is the most common. Resistance to ETEC F4ab/ F4ac adhesion in pigs has been shown to be inherited as an autosomal recessive trait. In previous studies the ETEC F4ab/F4ac receptor locus $(F 4 b c R)$ was mapped to the $\mathrm{q} 41$ region on pig chromosome 13. A polymorphism within an intron of the mucin 4 (MUC4) gene, which is one of the possible candidate genes located in this region, was shown earlier to cosegregate with the $F 4 b c R$ alleles. Recently, we
\end{abstract}

A. Rampoldi and M. J. Jacobsen contributed equally to this work.

A. Rampoldi · H. U. Bertschinger · D. Joller · P. Vögeli ( $₫)$.

S. Neuenschwander

Institute of Plant, Animal and Agroecosystem Sciences,

ETH Zurich, 8092 Zurich, Switzerland

e-mail: peter.voegeli@inw.agrl.ethz.ch

M. J. Jacobsen · M. Fredholm · C. B. Jørgensen Basic Animal and Veterinary Sciences, University of Copenhagen, 1870 Frederiksberg, Copenhagen, Denmark

E. Bürgi

Department of Farm Animals, University of Zurich, 8057 Zurich, Switzerland

L. Andersson

Department of Medical Biochemistry and Microbiology,

Uppsala University, 75123 Uppsala, Sweden

\section{A. L. Archibald}

Division of Genetics and Genomics, The Roslin Institute and R(D)SVS, University of Edinburgh, Roslin,

Midlothian EH25 9PS, UK discovered a Large White boar from a Swiss experimental herd with a recombination between $F 4 b c R$ and MUC4. A three-generation pedigree including 45 offspring was generated with the aim to use this recombination event to refine the localization of the $F 4 b c R$ locus. All pigs were phenotyped using the microscopic adhesion test and genotyped for a total of 59 markers. The recombination event was mapped to a $220-\mathrm{kb}$ region between a newly detected SNP in the leishmanolysin-like gene ( $L M L N$ g.15920) and SNP ALGA0072075. In this study the six SNPs ALGA0072075, ALGA0106330, MUC13-226, MUC13-813,DIA0000584, and MARC0006918 were in complete linkage disequilibrium with $F 4 b c R$. Based on this finding and earlier investigations, we suggest that the locus for $F 4 b c R$ is located between the $L M L N$ locus and microsatellite S0283.

\section{Introduction}

Close association to host surface is an essential requirement for successful induction of disease by a broad variety of pathogens. This association is often mediated by lectin recognition of host cell glycoconjugates (Karlsson 1998). Breeding for genetic resistance is an established routine in plant sciences and has become an attractive goal in animal sciences. Thus, selection for absence of receptor molecules or for genetic variants of receptor molecules to which the pathogen cannot bind would represent an attractive route to genetic resistance.

The two most economically dominant porcine enteric $E$. coli infections comprise diarrhea induced by diverse enterotoxins and enterotoxemia mediated by a shigatoxin. Both diseases have in common that the bacteria have to colonize the small intestine in high numbers where they produce the deleterious toxins. To build up huge 
populations, the bacteria are forced by the short transit time of ingesta to adhere to the small intestine's inner surface. This adhesion is the result of a highly specific interaction between fimbriae produced by the bacteria and receptors present exclusively on the brush border of small-intestine enterocytes (Bertschinger and Fairbrother 1999). Of the five pig-specific types of fimbriae, designated F4, F5, F6, F18, and F41 (Osek 1999), so far it is known that only two, F18 and F4, are highly prevalent. Antigen variants have been detected for both types of fimbriae. Whereas the two variants F18ab and F18ac recognize the same receptor (Rippinger et al. 1995), the three variants of F4 vary in their receptor specificities (Bijlsma et al. 1982). However, F4ac is by far the most prevalent variant all over the world with the exception of central China, where F4ad is reported to be predominant (Wang et al. 2006). Fortunately, the causative mutation for E. coli F18 susceptibility is known (Meijerink et al. 1997, 2000) and elimination of the E. coli F18 susceptibility allele from the porcine population is currently done in Switzerland and other countries to increase the number of resistant piglets in commercial populations (Luther et al. 2009).
In pigs the resistant and susceptible phenotypes for adherence or infection by $E$. coli with F4ac fimbriae are inherited as a monogenetic trait, the susceptibility allele being dominant over the resistance one (Gibbons et al. 1977). The receptor for F4ac or a closely linked locus binds F4ab as well (Jørgensen et al. 2003; Python et al. 2002). As yet, attempts to develop practical methods for identification of breeding stock that inherit resistance have not been completely successful. Several investigators have mapped the ETEC F4ab/F4ac receptor gene $(F 4 b c R)$ to SSC13 (Edfors-Lilja et al. 1995; Python et al. 2002). An overview of the marker positions and mapped genes encompassing the $F 4 b c R$ is given in Fig. 1. Subsequent studies mapped the ETEC F4ab/F4ac susceptibility locus to the interval $S W 207-S 0075$ refined in a $5.7-\mathrm{cM}$ region around the $F 4 b c R$ locus (Joller et al. 2009). Jørgensen et al. (2004) reported an association between resistance and a mutation in intron 7 of the mucin 4 (MUC4 g.8227 G > C) gene. However, data presented later by Rasschaert et al. (2007) as well as observations in Switzerland (Joller 2009) raised doubt as to whether this mutation in the MUC4 gene is in some rare cases not in complete linkage disequilibrium (LD) with the F4ac receptor locus.
Fig. 1 Location of 59 markers in the $F 4 b c R$ candidate region at SSC13. The gene order and the scale are deduced from the Sscrofa9 assembly. The positions of the microsatellites are shown to the right of the chromosome 13 idiogram. The approximate positions of candidate genes are indicated at the far right side. The number of markers in each gene is given in parenthesis. The position of the six SNPs ALGA0072075, ALGA0106330, MUC13-226, MUC13-813, DIA0000584, and MARC000691, which were in complete LD, are shown in bold

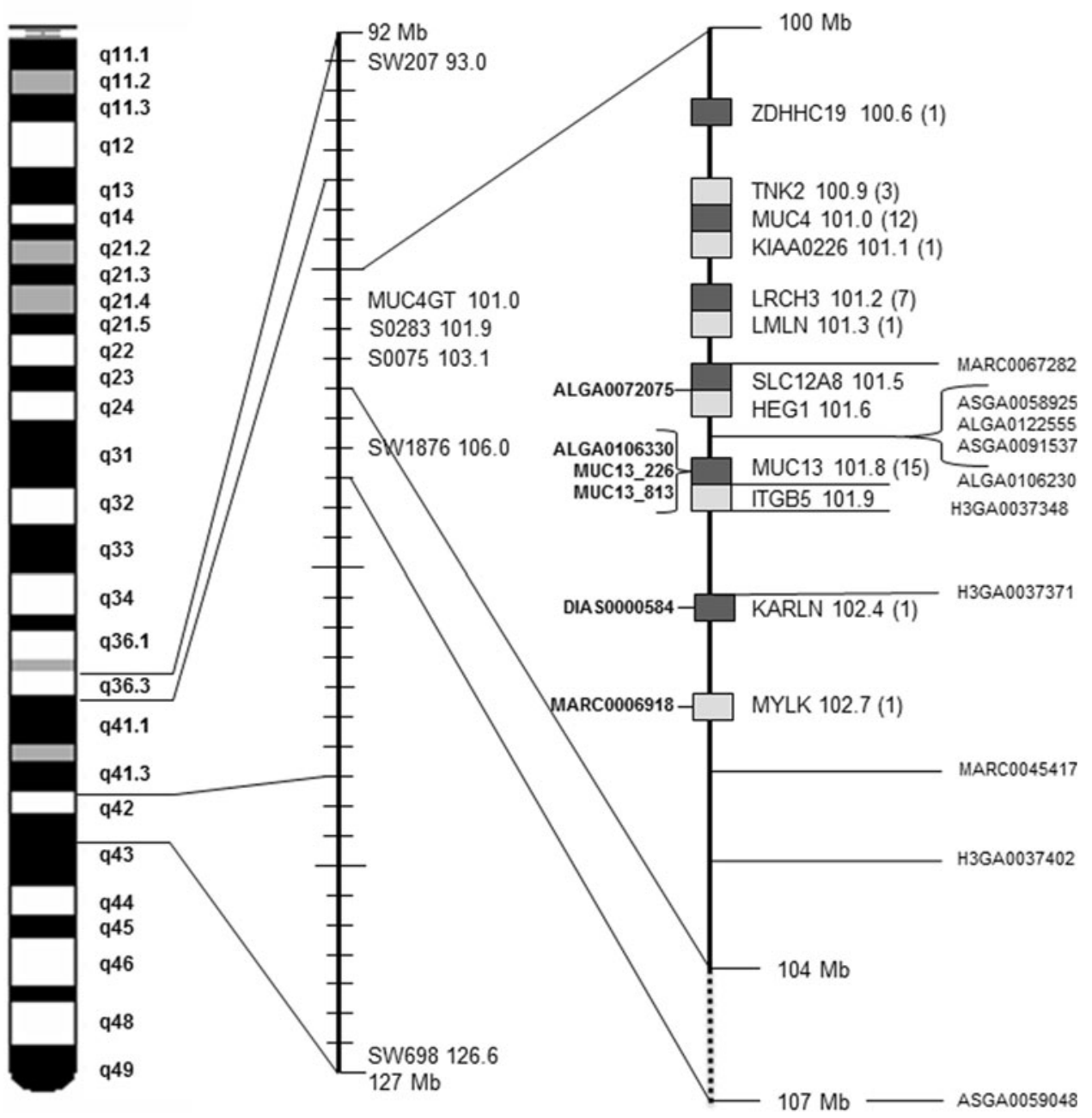


Fig. 2 Diagram of the three-generation family tree and haplotypes of eight pigs in the $F 4 b c R$ region. SNP names and positions on SSC13 are given at the right side. The animal identity is shown on top of the haplotypes. The SNP genotypes are depicted on the right side of the colored columns. The digit 1 corresponds to nucleotide A, 2 to $\mathrm{C}, 3$ to $\mathrm{G}$, and 4 to T. Microsatellite markers are represented by 5 , 6 , etc., depending on the sizes of the allelic bands. $S$ on top of the colored bars indicates the adhesive and $s$ the nonadhesive haplotype. Each haplotype has its own color. The lines indicate the relationships between the pigs. The geometric figures above the animal identity indicate the sex, squares for males and circles for females. Filled figures indicate $\mathrm{F} 4 \mathrm{ab} / \mathrm{F} 4 \mathrm{ac}$ susceptible and blank figures resistant pigs

We recently discovered a Large White boar that carried a recombinant chromosome in the interval between $F 4 b c R$ and MUC4. The aim of the study was to characterize the recombinant haplotype and also to discover more accurate markers for F4ab/F4ac resistance. Therefore, we tested a total of 59 markers (53 SNPs and 6 microsatellites) in a three-generation pedigree. The data indicate that the $F 4 b c R$ receptor locus is distal to the MUC4 locus.

\section{Materials and methods}

Pigs

The animals used in this study originated from a Swiss experimental herd (SEH) at the University of Zurich comprising Large White, Landrace purebred pigs, and Large White/Landrace crossbreds. The boar 698B used for breeding purposes was found to be homozygous for the susceptibility allele at the $F 4 b c R$ locus but heterozygous in ZDHHC19 g.4043, TNK2 g.7075, TNK2 g.7717, TNK2 g.11142, MUC4 g.8227, and KIAA0226 g.62250, which were so far in complete LD with the $F 4 b c R$ alleles (Jacobsen et al. 2010). To be sure that 698B was homozygous susceptible to ETEC F4ab/F4ac, it was mated to three resistant sows generating a total of 45 offspring. The homozygosity for the resistant allele of the sows was proved by previous matings to resistant boars and confirmed by the microscopic adhesion test after slaughtering.

To precisely define the location of the recombination event, a three-generation family was generated, including boar 698B, its parents (boar 381B and sow 405B), two siblings of boar 698B (9499 and 9486), and its offspring (1977, 2122, 2731, and 2734) (Fig. 2).

Sampling of intestinal tissue and microscopic adhesion test

Pigs $(n=53)$ were slaughtered at about 2 months of age, at 150 days of age, or when they were eliminated from

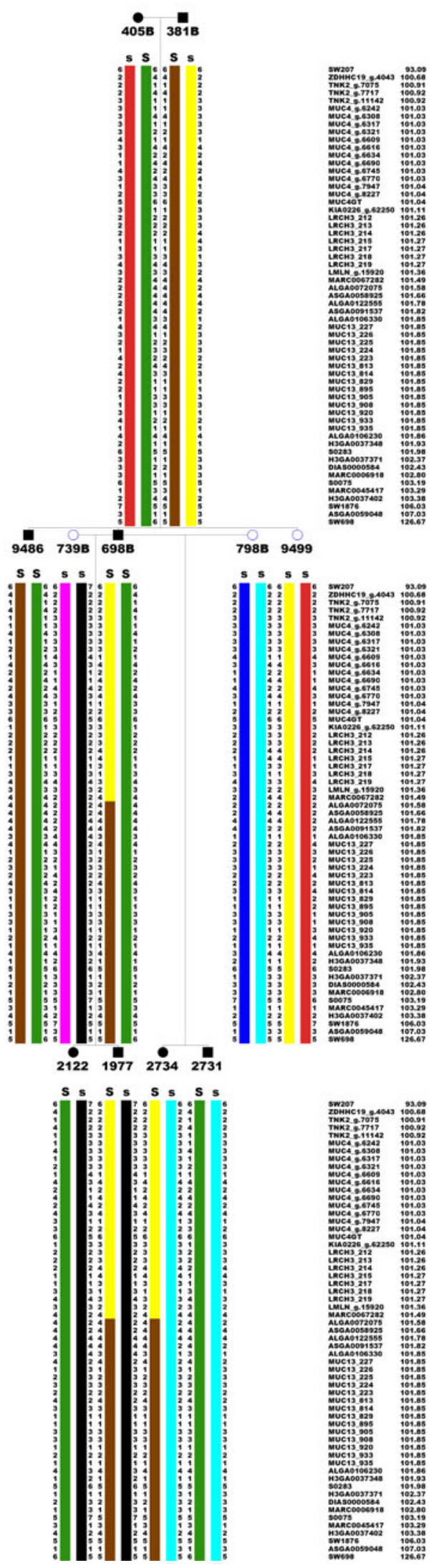


breeding. Samples of blood and intestine were taken at that time. The interval between killing and sampling of intestine was between 10 and 30 min to avoid degeneration of the epithelial intestinal cells.

All animals were phenotyped with the antigenic variant F4ac fimbriae by the microscopic adhesion test according to the method of Vögeli et al. (1996). A more detailed description of the preparation of bacterial strains, sampling of intestinal tissues, purification of enterocytes, and the procedure of the microscopic adhesion test can be found in Joller (2009). In brief, starting at the upper mesentery root, the intestine was separated from the mesenterium and an empty segment in the middle part was taken anywhere between 3.5 and $7.5 \mathrm{~m}$ in young pigs and between 5 and $10 \mathrm{~m}$ in older pigs. Twenty intact enterocytes were scored under an optical microscope by the same person. A pig was classified as adhesive to $\mathrm{F} 4 \mathrm{ac}$ if more than $15 \%$ of the enterocytes bound more than five bacteria on the brush borders.

\section{Screening for genetic polymorphisms}

Genomic DNA was extracted from EDTA-anticoagulated whole blood as described by Vögeli et al. (1994). A total of 59 markers on chromosome 13q41 were used to characterize the three-generation pedigree (53 SNPs listed in Tables 1 and 2) along with 6 microsatellite markers: SW207, MUC4GT, S0283, S0075, SW1876, and SW698. Twenty-nine markers from Table 1 were selected from Jacobsen et al. (2010), nine SNPs in MUC13 were taken from Zhang et al. (2008), and $L M L N$ g. 15920 was detected in this study by BLASTing the human sequence (NM_001136049) to the pig genome and by sequencing the genomic porcine homolog. The 14 SNPs listed in Table 2 were analyzed using the Illumina PorcineSNP60 BeadChip (Illumina, Inc., San Diego, CA, USA) (Ramos et al. 2009). The microsatellite markers were selected from Python et al. (2002).

PCR was carried out in a reaction volume of $25 \mu \mathrm{l}$ containing $100 \mathrm{ng}$ of genomic DNA, $10 \mathrm{mM}$ Tris-HCl (pH 9), $50 \mathrm{mM} \mathrm{KCl}, 1.5 \mathrm{mM} \mathrm{MgCl}_{2}, 200 \mu \mathrm{M}$ of each deoxynucleotide, $0.4 \mu \mathrm{M}$ of forward and reverse primers, and $1.25 \mathrm{U}$ of Taq DNA polymerase. The annealing temperature was chosen according to the melting temperature of the primers. For microsatellites, the PCR consisted of 25 cycles and for the SNPs 35 cycles followed by a final extension at $72^{\circ} \mathrm{C}$ for $2 \mathrm{~min}$. Thermal cycling conditions were incubation at $94^{\circ} \mathrm{C}$ for $2 \mathrm{~min}$ followed by incubation at the specific annealing temperature for $30 \mathrm{sec}$ and extension at $72^{\circ} \mathrm{C}$ for $30 \mathrm{sec}$. The subsequent denaturation was at $94^{\circ} \mathrm{C}$ for $30 \mathrm{sec}$. DNA samples were then purified with Montage PCR Centrifugal Filter Devices (p36461) (Millipore, Billerica, MA, USA) and sequenced. Alleles of the microsatellites were analyzed on an ABI377 DNA analyzer (Applied Biosystems, Foster City, CA, USA). Haplotypes were determined using MERLIN (Abecasis et al. 2002) and HaploPainter software (Thiele and Nürnberg 2005).

\section{Results}

The distribution of the 59 markers in the candidate region of the $F 4 b c R$ locus is shown in Fig. 1, and the approximate positions of genes and markers are according to the Sscrofa9 assembly and the physical map (http://pre. ensembl.org/Sus_scrofa_map/). These markers cover the 35-Mb interval from microsatellite SW207 to SW698. Fifty-five markers map to the core interval $100-104 \mathrm{Mb}$ on chromosome 13.

Boar B698 was mated with three sows resistant to ETEC $\mathrm{F} 4 \mathrm{ab} / \mathrm{F} 4 \mathrm{ac}$ and hence putative homozygotes for the absence of an adherent ETEC F4ab/F4ac receptor, generating a total of 45 offspring. All of the offspring were adhesive to ETEC F4ab/F4ac according to the microscopic adhesion test. Thus, all the offspring are presumed to have received (from boar B698) a susceptibility allele encoding the presence of a receptor to which $E$ coli $\mathrm{F} 4 \mathrm{ac}$ can adhere. Therefore, it can be deduced that boar B698 is homozygous for the susceptibility allele. However, the genotyping data showed that 23 piglets $(51.1 \%)$ possessed the MUC4 g.8227 C/C genotype, which was previously reported to be associated with the absence of F4ab/F4ac adhesion (Jacobsen et al. 2010; Jørgensen et al. 2004), and 22 piglets were $C / G(48.9 \%)$. Haplotype analysis of the genotype data reveals a putative recombination event between LMLM g.15920 and ALGA0072075 SNP marker loci (Fig. 2). The recombinant chromosome was transmitted to 23 of B698's offspring. The recombinant chromosome was identical to all other susceptible haplotypes for the interval ALGA0072075-SW698. The LMLN-S0075 region was also covered with 11 SNPs of the PorcineSNP60 BeadChip (Table 2), 2 microsatellites, and 14 SNPs in MUC13. In contrast to the MUC4 markers, ALGA0072075, ALGA0106330, MUC13-226, MUC13-813, DIA0000584, and MARC0006918 were all in complete LD with $F 4 b c R$ in our family (Fig. 2).

\section{Discussion}

Previous studies identified MUC4 as the most probable candidate gene for $F 4 b c R$ based on its chromosomal location and evidence that the molecule to which the $E$. coli F4ac adheres is a mucin-like sialoglycoprotein (Erickson et al. 1994; Jacobsen et al. 2010; Joller et al. 2009; Jørgensen et al. 2004). As reported earlier, the resistance 


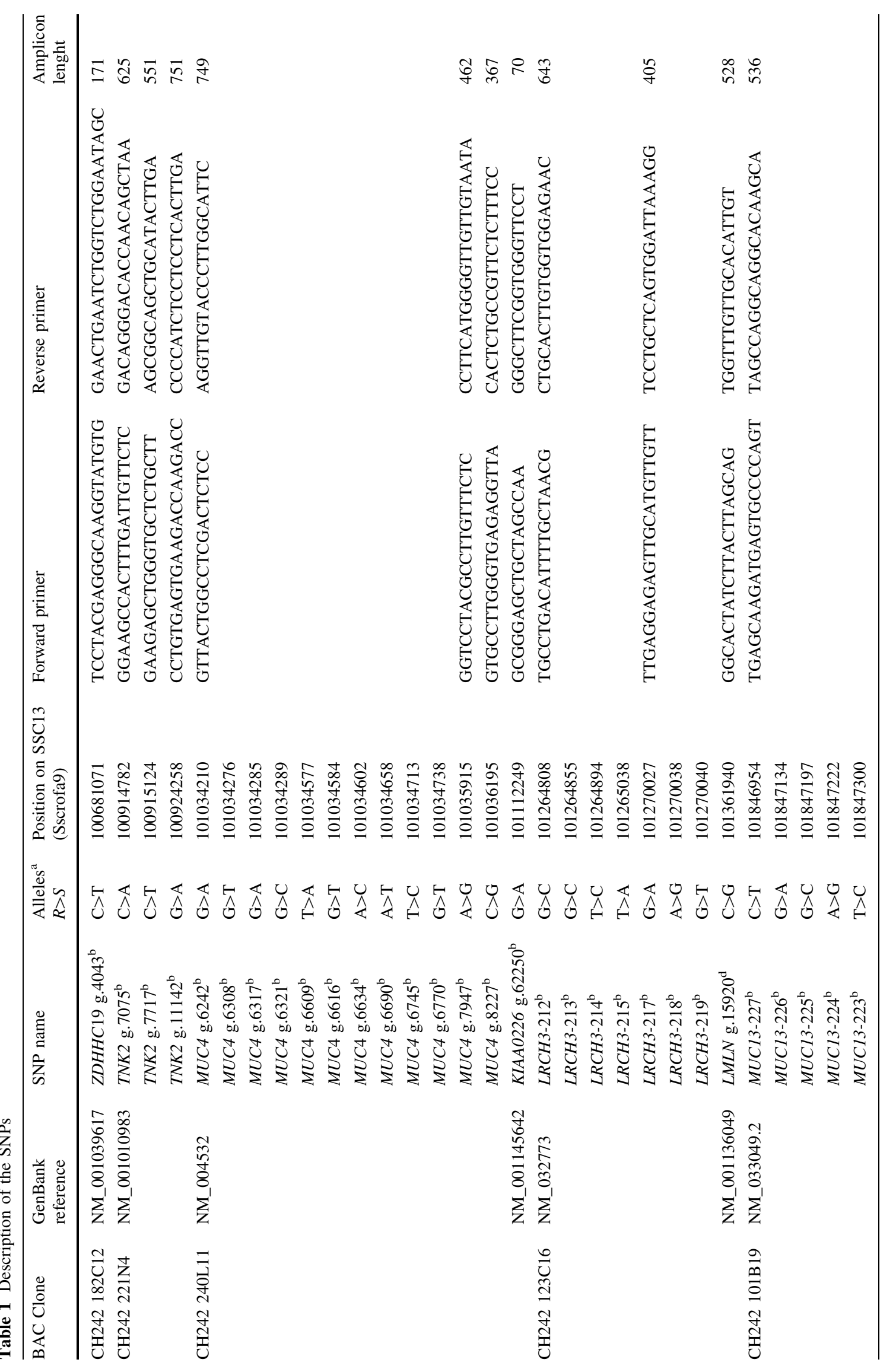




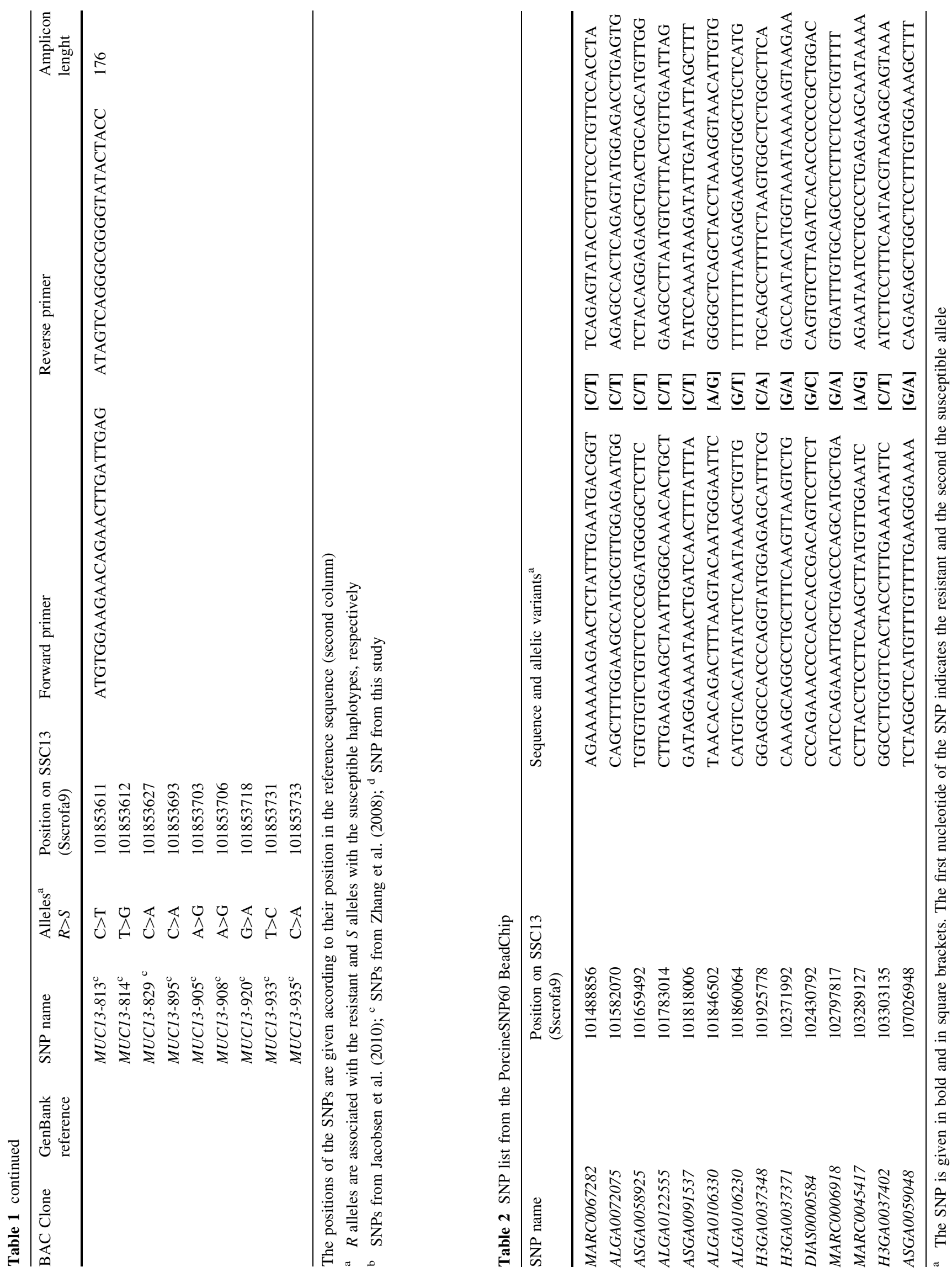


allele is presumed to represent the ancestral allele and is associated with greater haplotype heterogeneity (see 798B and 739B, Fig. 2) (Jacobsen et al. 2010). The large shared haplotype block associated with the susceptibility allele handicaps efforts to locate and identify the causative mutation. In addition, determination of the $F 4 b c R$ genotypes is time-consuming as it has to be done by progeny phenotyping which leads to the loss of potential breeding animals. Thus, when we detected a boar with a recombination between the MUC4 markers and $F 4 b c R$, it represented an opportunity to refine the search for the causative mutation. Therefore, we mated this boar to homozygous resistant sows and analyzed more markers distal to MUC4.

Boar 698B inherited a recombinant haplotype from its father, boar 381B. The recombinant allele was transmitted from 698B to half of its progeny, as seen in pigs 1977 and 2734 (Fig. 2), and was not found in other pigs from the same herd. The recombination in 698B occurred in an interval of about $220 \mathrm{~kb}$, between SNPs $L M L N$ g. 15920 and ALGA0072075. Boars 381B and 698B were found to be homozygous for the SNP MARC0067282, making it impossible to determine the position of the crossing over in 698B more precisely.

These results support the hypothesis that the locus for $F 4 b c R$ is located distal to MUC4. Besides the experimental pigs, we phenotyped 78 unrelated pigs of 38 litters of the Swiss performing station and determined their MUC4 g.8227 genotype (Joller 2009). Five pigs were phenotyped as resistant, whereas the genotype was MUC4 g.8227 $C / G$ and one susceptible pig was $M U C 4$ g. $8227 C / C$. These pigs were typed with the markers used in this study and the resistant pigs were homozygous A/A in ALGA0106330, $G / G$ in $M U C 13-226$, and $C / C$ in $M U C 13-813$, consistent with a resistant genotype. The susceptible pig was heterozygous $A / G, A / G$, and $C / T$, respectively, for the same markers. These results suggest stronger LD between these markers and F4bcR than between MUC4 g.8227 and F4bcR and give further evidence that $F 4 b c R$ is located distal to MUC4.

BLAST analyses of the markers that were in complete LD with $F 4 b c R$ showed that only MUC13-813 was located in an exon. However, this $\mathrm{C}>\mathrm{T}$ transition is silent. ALGA0072075 was mapped between SLC12A8 and HEG1. ALGA0106330 was located in an intron of MUC13. DIAS0000584 was mapped to an intron of gene KALRN and MARC0006918 to an intron of MYLK. These findings indicate that these markers may not be causative for ETEC F4ab/F4ac adhesion.

Joller et al. (2006) had initially mapped the most probable position of the F4bcR locus in the SW207-S0283 interval. Unfortunately, the family in this study did not allow the identification of a distal border closer to the unknown F4bcR than microsatellite S0283. Therefore, we propose the interval between $L M L N$ g.15920 and S0283, consisting of around $620 \mathrm{~kb}$, to be the most probable region for $F 4 b c R$.

Mucin-type proteins and lipids are present on almost every epithelial tissue and are important for defense against pathogens. However, mucins and other glycosylated structures are also targets for microbial attachment as they contain a variety of oligosaccharide structures providing binding sites for bacteria. In the case of bacteria expressing the F18-type fimbriae, the adhesion is dependent on the activity of fucosyltransferase 1 and the presence of a yet unknown fucosylated structure (Meijerink et al. 2000). Although the causative mutation in F4ac is still unknown, the genetic analyses performed in 698B's family have determined that the causal mutation for ETEC F4ab/F4ac susceptibility is downstream of the gene $L M L N$ and probably may be located around the region containing the MUC13 gene.

Acknowledgments We thank Prof. Martien Groenen and his group (Animal Breeding and Genetics, Wageningen University, Wageningen, The Netherlands) for providing the SNP sequences and their positions in the Sscrofa9 assembly. We thank Michael Goe for editing the manuscript and the Genomic Diversity Center (ETH Zurich) for allowing us to use their infrastructure. The study was supported by the Swiss National Science Foundation (grant No. 3100A0-120255/1), by the ETH Zurich, and the SUISAG, Sempach (Dr. Andreas Hofer).

\section{References}

Abecasis GR, Cherny SS, Cookson WO, Cardon LR (2002) Merlinrapid analysis of dense genetic maps using sparse gene flow trees. Nat Genet 30:97-101

Bertschinger HU, Fairbrother JM (1999) Escherichia coli infections. In: Straw BE, D'Allaire S, Mengeling WL, Taylor DJ (eds) Diseases of Swine, 8th edn. Iowa State University Press, Ames, IA, pp 431-468

Bijlsma IG, de Nijs A, van der Meer C, Frik JF (1982) Different pig phenotypes affect adherence of Escherichia coli to jejunal brush borders by K88ab, K88ac, or K88ad antigen. Infect Immun 37:891-894

Edfors-Lilja I, Gustafsson U, Duval-Iflah Y, Ellergren H, Johansson M et al (1995) The porcine intestinal receptor for Escherichia coli K88ab, K88ac: regional localization on chromosome 13 and influence of $\mathrm{IgG}$ response to the K88 antigen. Anim Genet $26: 237-242$

Erickson AK, Baker DR, Bosworth BT, Casey TA, Benfield DA et al (1994) Characterization of porcine intestinal receptors for the K88ac fimbrial adhesion of Escherichia coli as mucin-type sialoglycoproteins. Infect Immun 62:5404-5410

Gibbons RA, Sellwood R, Burrows M, Hunter PA (1977) Inheritance of resistance to neonatal E. coli diarrhoea in the pig: Examination of the genetic system. Theor Appl Genet 51:65-70

Jacobsen M, Kracht SS, Esteso G, Cirera S, Edfors I et al (2010) Refined candidate region specified by haplotype sharing for Escherichia coli $\mathrm{F} 4 \mathrm{ab} / \mathrm{F} 4 \mathrm{ac}$ susceptibility alleles in pigs. Anim Genet 41:21-25

Joller D (2009) Comparative molecular approaches to identify host determinants mediating adhesion of $E$. coli $\mathrm{F} 4$ strains in pigs. 
Dissertation ETH Zürich number 18518. Available at http:// e-collection.ethbib.ethz.ch/eserv/eth:445/eth-445-02.pdf\#search= $\%$ 22Joller\%20David\%22

Joller D, Jorgensen CB, Bertschinger HU, Bürgi E, Stannarius C et al (2006) Refined linkage mapping of the Escherichia coli F4ac receptor gene on pig chromosome 13. Proceedings of the 30th International Conference for Animal Genetics, Porto Seguro, Brazil, August 20-25, 2006

Joller D, Jorgensen CB, Bertschinger HU, Python P, Edfors I et al (2009) Refined localization of the Escherichia coli F4ab/F4ac receptor locus on pig chromosome 13. Anim Genet 40:749-752

Jørgensen CB, Cirera S, Archibald AL, Anderson L, Fredholm M et al (2004) Porcine polymorphisms and methods for detecting them. International application published under the patent cooperation treaty (PCT), WO2004/048606A2

Jørgensen CB, Cirera S, Anderson SI, Archibald AL, Raudsepp T et al (2003) Linkage and comparative mapping of the locus controlling susceptibility towards E. coli F4ab/ac diarrhoea in pigs. Cytogenet Genome Res 102:157-162

Karlsson KA (1998) Meaning and therapeutic potential of microbial recognition of host glycoconjugates. Mol Microbiol 29:1-11

Luther H, Vögeli P, Hofer A (2009) Increasing genetic E.coli F18 resistance in Swiss pigs. Available at http://www.eaap.org/ Barcelona/Book_Abstracts.pdf

Meijerink E, Fries R, Vögeli P, Masabanda J, Wigger G et al (1997) Two alpha $(1,2)$ fucosyltransferase genes on porcine chromosome $6 \mathrm{q} 11$ are closely linked to the blood group inhibitor (S) and Escherichia coli F18 receptor (ECF18R) loci. Mamm Genome 8:736-741

Meijerink E, Neuenschwander S, Fries R, Dinter A, Bertschinger HU et al (2000) A DNA polymorphism influencing alpha(1, 2)fucosyltransferase activity of the pig FUT1 enzyme determines susceptibility of small intestinal epithelium to Escherichia coli F18 adhesion. Immunogenetics 52:129-136

Osek J (1999) Prevalence of virulence factors of Escherichia coli strains isolated from diarrheic and healthy piglets after weaning. Vet Microbiol 68:209-217
Python P, Jorg H, Neuenschwander S, Hagger C, Stricker C et al (2002) Fine-mapping of the intestinal receptor locus for enterotoxigenic Escherichia coli $\mathrm{F} 4 \mathrm{ac}$ on porcine chromosome 13. Anim Genet 33:441-447

Ramos AM, Crooijmans RP, Affara NA, Amaral AJ, Archibald AL et al (2009) Design of a high density SNP genotyping assay in the pig using SNPs identified and characterized by next generation sequencing technology. PLoS One 4:e6524

Rasschaert K, Verdonck F, Goddeeris BM, Duchateau L, Cox E (2007) Screening of pigs resistant to F4 enterotoxigenic Escherichia coli (ETEC) infection. Vet Microbiol 123:249-253

Rippinger P, Bertschinger HU, Imberechts H, Nagy B, Sorg I et al (1995) Designations F18ab and F18ac for the related fimbrial types F107, 2134P and 8813 of Escherichia coli isolated from porcine postweaning diarrhoea and from oedema disease. Vet Microbiol 45:281-295

Thiele H, Nürnberg P (2005) HaploPainter: a tool for drawing pedigrees with complex haplotypes. Bioinformatics 21:1730-1732

Vögeli P, Bolt R, Fries R, Stranzinger G (1994) Co-segregation of the malignant hyperthermia and the Arg615-Cys615 mutation in the skeletal muscle calcium release channel protein in five European Landrace and Pietrain pig breeds. Anim Genet 25 Suppl 1:59-66

Vögeli P, Bertschinger HU, Stamm M, Stricker C, Hagger C et al (1996) Genes specifying receptors for F18 fimbriated Escherichia coli, causing oedema disease and postweaning diarrhoea in pigs, map to chromosome 6. Anim Genet 27:321-328

Wang J, Jiang SW, Chen XH, Liu ZL, Peng J (2006) Prevalence of fimbrial antigen (K88 variants, K99 and 987P) of enterotoxigenic Escherichia coli from neonatal and post-weaning piglets with diarrhea in central China. Asian Austral J Anim Sci 19(9):1342-1346

Zhang B, Ren J, Yan X, Huang X, Ji H et al (2008) Investigation of the porcine $M U C 13$ gene: isolation, expression, polymorphisms and strong association with susceptibility to enterotoxigenic Escherichia coli F4ab/ac. Anim Genet 39:258-266 